\title{
Relationship between common interleukin 1-beta gene polymorphisms and the risk of gestational disorders: An updated meta-analysis
}

\author{
Mahdiyeh Harati-Sadegh ${ }^{1}$, Saman Sargazi ${ }^{* 2}$ (D) Hamed Taheri $^{2,3}$, Narges Arbabi ${ }^{4}$, Ramin Saravani*2,5 (D), \\ Shekoufeh Mirinejad²
}

Received: 11 Apr 2020

Published: 17 Feb 2021

\begin{abstract}
Background: To quantitatively estimate the relationship between IL-1 $\beta-511 \mathrm{C}>\mathrm{T},-31 \mathrm{~T}>\mathrm{C}$, and $+3954 \mathrm{C}>\mathrm{T}$ polymorphisms and risk of gestational disorders.

Methods: In this meta-analysis, eligible publications were searched in Web of Knowledge, MEDLINE, PubMed, Scopus, and Google Scholar databases (updated April 2020), using appropriate or relevant keywords. Case-control population-based reports were included if provided with genotypic frequencies of both studied groups. Statistical analyses were performed using the MetaGenyo web tool software, where a $\mathrm{P}$ value less than 0.05 indicated a significant association. For the assessment of between-study variations, heterogeneity analysis was applied with the $\mathrm{I}^{2}$ statistics.

Results: A total of thirteen studies were included. We observed a significant association between IL-1 $\beta-31 \mathrm{~T}>\mathrm{C}$ polymorphism and reduced risk of gestational disorders under codominant $\mathrm{CT}$ vs. $\mathrm{CC}[\mathrm{OR}=0.74, \mathrm{CI}(0.59-0.92)]$, and dominant $\mathrm{CT}+\mathrm{TT}$ vs. $\mathrm{CC}$ [OR= 0.74 , CI (0.60-0.91)] contrasted genetic models. The stratified analysis considering the disease type showed that the 511C $>\mathrm{T}$ variant, under the recessive CC vs. CT+TT model, enhanced the risk of preterm birth by 1.29 fold.

Conclusion: Our results failed to support an association between two IL-1 $\beta$ polymorphisms, $511 \mathrm{C}>\mathrm{T}$ and $+3954 \mathrm{C}>\mathrm{T}$, with the overall risk of gestational disorders. In contrast, the $31 \mathrm{~T}>\mathrm{C}$ variant reduced the incidence of such diseases. Further studies are encouraged to get more precise estimates of effect sizes.
\end{abstract}

Keywords: Cytokine, Interleukin, Polymorphism, Pregnancy, Meta-analysis

Conflicts of Interest: None declared

Funding: None

*This work has been published under CC BY-NC-SA 1.0 license.

Copyright $\odot$ Iran University of Medical Sciences

Cite this article as: Harati-Sadegh M, Sargazi S, Taheri H, Arbabi N, Saravani R, Mirinejad Sh. Relationship between common interleukin 1-beta gene polymorphisms and the risk of gestational disorders: An updated meta-analysis. Med J Islam Repub Iran. 2021 (17 Feb);35:25. https://doi.org/10.47176/mjiri.35.25

\section{Introduction}

Gestational disorders are a heterogeneous group of conditions affecting pregnant women with unknown etiology

Corresponding author: Dr Saman Sargazi, sgz.biomed@zaums.ac.ir

Dr Ramin Saravani, saravaniramin@zaums.ac.ir

1. Genetic of Non-Communicable Disease Research Center, Resistant Tuberculosis Institute, Zahedan University of Medical Sciences, Zahedan, Iran

2. Cellular and Molecular Research Center, Resistant Tuberculosis Institute, Zahedan University of Medical Sciences, Zahedan, Iran

3. Department of Internal Medicine, School of Medicine, Zahedan University of Medical Sciences, Zahedan, Iran

4. Infectious Diseases and Tropical Medicine Research Center, Resistant Tuberculosis Institute, Zahedan University of Medical Sciences, Zahedan, Iran

5. Department of Clinical Biochemistry, School of Medicine, Zahedan University of Medical Sciences, Zahedan, Iran
$(1,2)$. Notwithstanding, many of these disorders are caused by insufficient oxygen transfer and/or nutrients

$\uparrow$ What is "already known" in this topic:

Gestational disorders are a heterogeneous group of conditions affecting pregnant women with unknown etiology. Regulation of inflammatory responses seems to be etiologically crucial in the pathogenesis of gestational diseases. Previous studies have shown the correlation between IL-1 $\beta$ gene polymorphisms and the risk of gestational disorders; still, the results showed inconsistency.

\section{$\rightarrow$ What this article adds:}

In this study, we observed a significant association between IL-1 $\beta-31 \mathrm{~T}>\mathrm{C}$ polymorphism and reduced risk of gestational disorders under $\mathrm{CT}$ vs. $\mathrm{CC}$ and $\mathrm{CT}+\mathrm{TT}$ vs. $\mathrm{CC}$ genetic contrasted models. Our results failed to support an association between the other two IL- $1 \beta$ polymorphisms, $511 \mathrm{C}>\mathrm{T}$ and $+3954 \mathrm{C}>\mathrm{T}$, with the overall risk of gestational disorders. 
from mother to the fetus $(3,4)$. Pregnancy itself is marked by increased expression of inflammatory mediators, such as chemokines, cytokines, and pattern recognition receptors, which is often occurred during gestation $(5,6)$. During gestation, the balance between multiple cytokines is profoundly altered. This might trigger the production of inflammatory cytokines, which are mainly involved in adverse pregnancy outcomes (7). Through innate immune response, an organism reacts to possible perturbations in organ function, playing a substantial role in preterm birth (PTB), preeclampsia, recurrent spontaneous abortion (RSA), etc. $(7,8)$. Moreover, high levels of inflammatory mediators were detected in placentae affected by gestational diabetes, causing defective placentation (9). That is to say, several types of gestational disorders share a mutual phenotype of variability in inflammatory responses.

Cytokines are immunomodulatory proteins that are primarily involved in many aspects of pregnancy (10). As a multifunctional cytokine, interleukin 1 (IL1) is produced by villous syncytiotrophoblast of the human placenta during early pregnancy $(11,12)$.

Several genetic variants of the IL-1 gene contribute to altered IL-1 expression, causing impaired embryogenesis and abortion (13). In this regard, single-nucleotide polymorphisms (SNPs) located within coding or noncodingregions of the IL-1 $\beta$ gene are well-studied. One wellstudied variant is rs16944, a C-to-T substitution variant that resided in the promoter region of the IL- $1 \beta$ gene. The other IL-1 $\beta$ variant, rs1143634 (in the +3954 locus), is associated with an elevated cytokine production as a fourfold higher amount of IL- $1 \beta$ was observed in homozygous carriers of the $\mathrm{T}$ allele compared with the CC genotype carriers (14). Besides, rs1143627 is a TATA box polymorphism (genotype T/C) in the -31 locus of the IL-1 $\beta$ gene (15). These SNPs are high-risk candidates, and investigating their effects will provide new information in developing novel treatment strategies for subjects with etiologically different conditions.

Regulation of inflammatory responses seems to be etiologically crucial in the pathogenesis of gestational disorders. Previous studies have established a link between IL-1 $\beta$ gene polymorphisms and the risk of gestational disorders (16-18). However, the results showed inconsistency. In this comprehensive study, we sought to explore a more accurate estimation of the relationship between three common IL-1 $\beta$ polymorphisms and susceptibility to gestational disorders.

\section{Methods}

\section{Study selection}

In this meta-analysis, we searched Web of Knowledge, MEDLINE, PubMed, Scopus, and Google Scholar databases for relevant reports (the last search on April 2020) and statistically combined the results of previous casecontrol studies. Keywords in the searches included "rs1143627 or IL-1 $\beta$-31T or rs16944 or IL-1 $\beta-511 \mathrm{~T}$ or rs 1143634 or IL-1 $\beta-3954 \mathrm{C} / \mathrm{T}$ " and "gestation or gestational or pregnancy or labor or miscarriage or abortion or preterm or preterm or preeclampsia or preeclampsia" and "polymorphism or mutation or variant or SNP or single- nucleotide polymorphism." Also, additional eligible studies were identified by the use of hand searching of retrieved articles.

High-quality case-control population-based reports were included if provided with genotypic frequencies of both studied groups. Cohort studies, review articles, conference abstracts, publication in other languages, low-quality studies, and duplicated data were excluded. If more than one different case-controls were reported in the same article, they were treated as independent studies.

\section{Data extraction}

Information including the first author's name, publication date, country, ethnicity, number of subjects in the studied groups, genotyping method, and the genotypic distribution of IL-1 $\beta$ polymorphisms $(-31 \mathrm{~T},-511$, and 3954 ) in cases and controls were recorded from each publication. Data extraction was done independently by two authors (S.S. and M.H.S.) from all eligible publications. In case of disagreement, a third reviewer (N.A.) settled the discrepancy.

\section{Risk of bias}

The quality assessment (QA) was performed according to the Newcastle-Ottawa Scale (NOS) as described previously (19), and scores are presented in Tables 1-3. Studies with a score $\leq 6$ were regarded as "low-quality," whereas those scored $>6$ were considered "high-quality."

\section{Statistical analysis}

Data were analyzed using the MetaGenyo web tool (20) and Stata v.12 software. The Mantel-Haenszel method was used to pool Odds Ratios (ORs) with $95 \%$ confidence intervals (CIs) for estimation of the strengths of association under the allelic and different genetic contrasted models (21). Subgroup analysis was performed by disease type, ethnicity, and score of each study. For each study, deviations from Hardy-Weinberg equilibrium (HWE in the controls) were checked using the Chi-square test. Heterogeneity analysis was applied with the $\mathrm{I}^{2}$ statistics ( $>50 \%$ as heterogeneity) to assess variations between studies. When appropriate, the fixed-effect model (in the absence of between-study variation) or the random effect model (in the presence of between-study variation) was applied to pool the data from individual studies. Publication bias was estimated using Egger's tests. Analysis of sensitivity was also performed to assess the robustness of summary ORs.

\section{Results}

\section{Study characteristics}

Upon performing a comprehensive literature search, 22 reports of genetic association studies were identified regarding three IL-1 $\beta$ polymorphisms and the risk of gestational disorders $(16-18,22-39)$ containing 7574 subjects (for IL-1 $\beta$-511), 2452 subjects (for IL-1 $\beta$-31), and 3092 subjects (for IL-1 $\beta$-3954). Eleven of these studies were performed on Asians, and eight studies were on Caucasians. Figure 1 shows the PRISMA diagram of the searching procedure. The characteristics of all included studies 


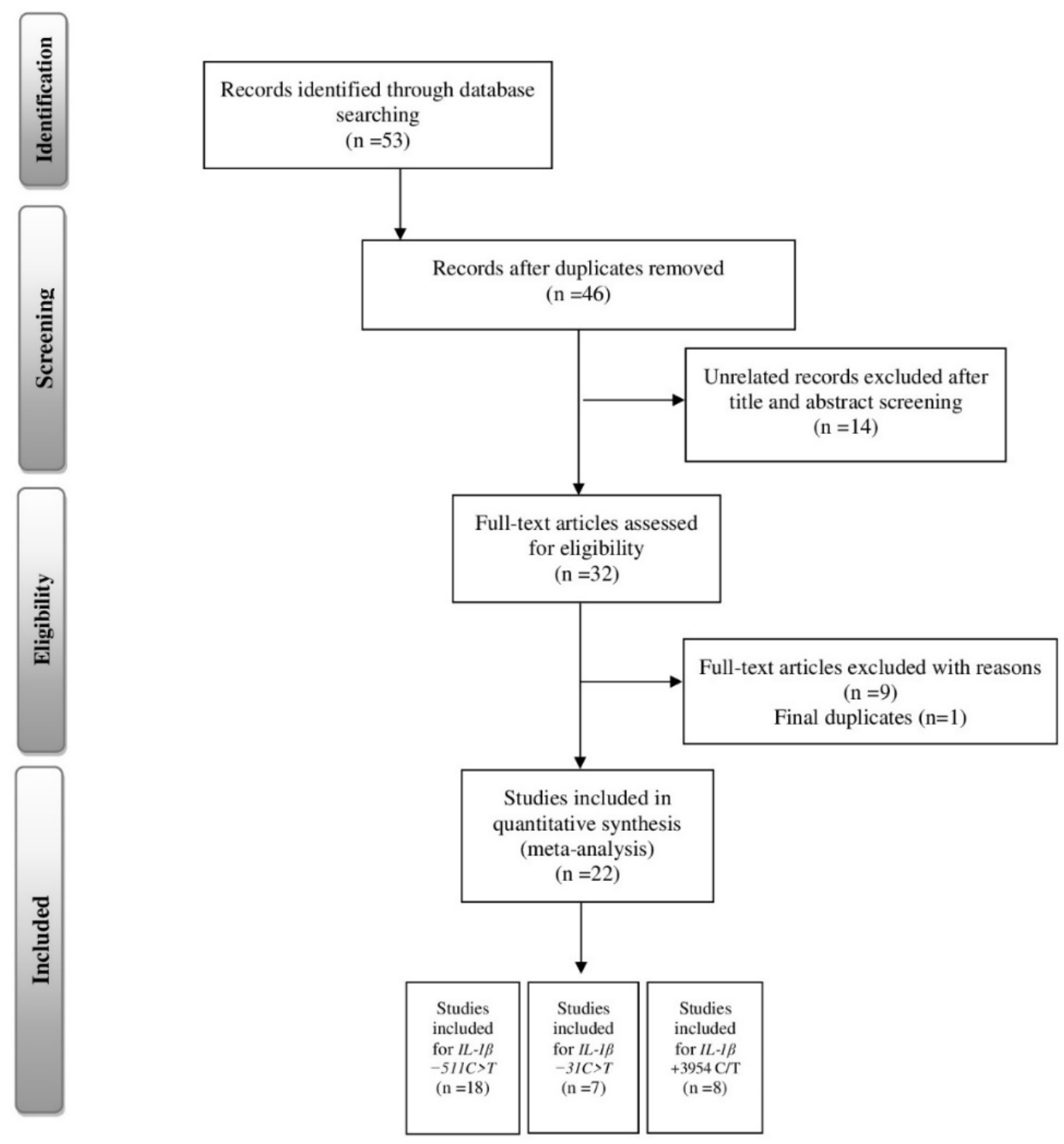

Fig. 1. Flow diagram of the study selection procedure

are summarized in Tables 1, 2, and 3.

Eight studies rated low-quality and therefore excluded. Finally, a total of 13 studies were included in the metaanalysis.

\section{Meta-analysis results of IL1-6 -511C >T polymorphism}

Eleven studies with a total of 2747 women with gestational disorders and 3436 controls were included to examine the correlation of IL1- $\beta-511 \mathrm{C}>\mathrm{T}$ polymorphism and gestational disorders risk. The Meta-analysis identified no significant link between the overall risk of gestational disorders and the SNP under different genetic models (Table 4). Subgroup analysis indicated an increased PTB risk under the recessive (OR 1.29, 95\% CI 1.06$1.58, \mathrm{p}=0.01$; $\mathrm{CC}$ vs. CT+TT) genetic model. Besides, stratified analysis by ethnicity revealed that this polymorphism conferred a protective effect against susceptibility to gestational disorders under the heterozygous codominant model in Asians (OR 0.85, 95\% CI 0.72-

$$
0.99, \mathrm{p}=0.036 ; \text { CT vs. TT) (Table 5). }
$$

\section{Meta-analysis results of IL1-6 -31T>C polymorphism}

By pooling the results of 5 studies, including 660 cases and 1433 healthy women, we found a noteworthy link between IL-1 $\beta-31 \mathrm{~T}>\mathrm{C}$ polymorphism and reduced the risk of gestational disorders under heterozygous codominant (OR $0.74,95 \%$ CI $0.59-0.92, \mathrm{p}=0.01$; CT vs. CC) and dominant (OR 0.74, 95\% CI 0.60-0.91, $\mathrm{p}=0.01$; CT+TT vs. CC) models (Table 4). Stratified analysis by ethnicity revealed a significant decrease in the risk of gestational disorders under codominant (OR 0.70, 95\% CI 0.55-0.89, $\mathrm{p}=0.003$; $\mathrm{CT}$ vs. CC) and dominant (OR 0.70 , 95\% CI 0.56$0.88, p=0.002$; CT+TT vs. CC) contrasted models. In this regard, no remarkable association was noticed in Caucasian women (Table 5). Figure 2 shows the forest plot for the association between the IL-1 $\beta-31 \mathrm{~T}>\mathrm{C}$ polymorphism and overall risk of gestational disorders under the codominant heterozygous CT vs. CC contrasted model. 
Table 1. Main characteristics of the studies included in the meta-analysis for IL-1 $\beta-511 \mathrm{C}>\mathrm{T}$

\begin{tabular}{|c|c|c|c|c|c|c|c|c|c|c|c|c|c|c|c|c|c|c|c|}
\hline Study (year) & Genotyping method & Disease & Country & Ethnicity & Case & Control & & & Cases & & & & & Control & & & $\mathrm{P}_{\text {HWE }}$ & $\mathrm{P}_{\mathrm{HWE}}$ & Score \\
\hline IL-1 $\beta-511 \mathrm{C}>\mathrm{T}$ & & & & & & & $\mathrm{CC}$ & CT & TT & $\mathrm{C}$ & $\mathrm{T}$ & $\mathrm{CC}$ & $\mathrm{CT}$ & TT & $\mathrm{C}$ & $\mathrm{T}$ & & & \\
\hline Hefler et al. (2002) & PCR-RFLP & RSA & Austria & Caucasian & 130 & 67 & 29 & 90 & 11 & 148 & 112 & 20 & 38 & 9 & 78 & 56 & 0.17 & 0.52 & 5 \\
\hline Wang et al. (2002) & PCR-RFLP & RSA & USA & Caucasian & 131 & 72 & 65 & 49 & 17 & 179 & 83 & 21 & 32 & 19 & 74 & 70 & 0.35 & 0.53 & 5 \\
\hline Linjawi et al. (2005) & PCR-RFLP & RSA & UK & Caucasian & 206 & 224 & 69 & 117 & 20 & 255 & 157 & 85 & 110 & 29 & 280 & 168 & 0.48 & 0.57 & 6 \\
\hline Sata et al. (2009) & RT PCR & РТВ & Japan & Asian & 73 & 341 & 26 & 27 & 20 & 79 & 67 & 86 & 162 & 93 & 334 & 348 & 0.36 & 0.45 & 8 \\
\hline Ma et al. (2011) & PCR-RFLP & RSA & China & Asian & 162 & 156 & 38 & 84 & 40 & 160 & 164 & 46 & 84 & 26 & 176 & 136 & 0.24 & 0.45 & 7 \\
\hline Agrawal et al. (2012) & PCR-RFLP & RSA & India & Asian & 200 & 300 & 13 & 86 & 101 & 112 & 288 & 15 & 126 & 159 & 156 & 411 & 0.11 & 0.51 & 7 \\
\hline Schmid et al. (2012) & PCR and pyrosequencing & РТВ & Austria & Caucasian & 100 & 100 & 37 & 47 & 16 & 121 & 79 & 43 & 39 & 18 & 125 & 75 & 0.09 & 0.37 & 6 \\
\hline Yilmaz et al. (2012) & PCR-RFLP & PTB & Turkey & Caucasian & 100 & 101 & 41 & 52 & 7 & 134 & 66 & 35 & 36 & 30 & 106 & 96 & 0.004 & 0.05 & 6 \\
\hline Kim et al. (2014) & PCR-RFLP & RSA & Korea & Asian & 385 & 232 & 96 & 190 & 99 & 382 & 388 & 39 & 120 & 73 & 198 & 266 & 0.38 & 0.45 & 7 \\
\hline Wang et al. (2014) & PCR-RFLP & PE & China & Asian & 232 & 447 & 52 & 98 & 82 & 202 & 262 & 125 & 214 & 108 & 464 & 430 & 0.38 & 0.45 & 7 \\
\hline Awasthi et al. (2015) & PCR-RFLP & PTB & India & Asian & 559 & 559 & 199 & 223 & 137 & 621 & 497 & 166 & 266 & 127 & 598 & 520 & 0.30 & 0.45 & 7 \\
\hline Langmia et al. (2016) & Mass ARRAY & РТВ & Malaysia & Asian & 92 & 391 & 22 & 49 & 21 & 93 & 91 & 104 & 203 & 84 & 411 & 371 & 0.42 & 0.45 & 7 \\
\hline Pereyra et & RT PCR & PTB & Uruguay & Hispanic & 142 & 100 & 44 & 81 & 17 & 169 & 115 & 23 & 61 & 16 & 107 & 93 & 0.02 & 0.14 & 7 \\
\hline Nasr et al. (2017) & PCR-RFLP & PE & Egypt & Arab & 80 & 80 & 17 & 32 & 31 & 66 & 94 & 24 & 40 & 16 & 88 & 72 & 0.93 & 0.93 & 6 \\
\hline Rahmani et al. (2018) & PCR-RFLP & RSA & Iran & Caucasian & 100 & 100 & 35 & 43 & 22 & 113 & 87 & 40 & 42 & 18 & 122 & 78 & 0.24 & 0.45 & 7 \\
\hline Wang et al. (2019) & PCR-RFLP & RSA & China & Asian & 598 & 603 & 149 & 294 & 155 & 592 & 604 & 110 & 275 & 218 & 495 & 711 & 0.16 & 0.45 & 8 \\
\hline Majcher et al. (2019) & Taqman & GDM & Poland & Caucasian & 204 & 207 & 93 & 93 & 18 & 279 & 129 & 86 & 95 & 26 & 267 & 147 & 0.98 & 0.98 & 7 \\
\hline
\end{tabular}

RSA: Recurrent spontaneous abortion; PE: Preeclampsia; PTB: Pre term birth; GDM: Gestational diabetes mellitus; UK: United Kingdom; USA: United States; RT PCR: Real-time PCR. PHwE: The P-value of chi-square test for Hardy-Weinberg in controls; PHWE: PHWE corrected for multiple testing by FDR method.

Table 2. Main characteristics of the studies included in the meta-analysis for IL-1 $\beta-31 \mathrm{~T}>\mathrm{C}$

\begin{tabular}{|c|c|c|c|c|c|c|c|c|c|c|c|c|c|c|c|c|c|c|c|}
\hline Study (year) & Genotyping & Disease & Country & Ethnicity & Case & Control & & & Cases & & & & & Control & & & $\mathrm{P}_{\mathrm{HWE}}$ & $\mathrm{P}_{\mathrm{HWE}^{*}}$ & Score \\
\hline IL- $1 \beta-31 \mathrm{~T}>\mathrm{C}$ & & & & & & & $\mathrm{CC}$ & $\mathrm{CT}$ & TT & $\mathrm{C}$ & $\mathrm{T}$ & $\mathrm{CC}$ & CT & TT & $\mathrm{C}$ & $\mathrm{T}$ & & & \\
\hline Wang et al. (2002) & PCR-RFLP & RSA & USA & Caucasian & 127 & 72 & 19 & 47 & 61 & 85 & 155 & 19 & 33 & 20 & 71 & 73 & 0.48 & 0.87 & 5 \\
\hline Sata et al. (2009) & RT PCR & PTB & Japan & Asian & 73 & 341 & 17 & 30 & 26 & 64 & 82 & 91 & 166 & 84 & 348 & 334 & 0.63 & 0.89 & 8 \\
\hline Ma et al. (2011) & PCR-RFLP & RSA & China & Asian & 162 & 156 & 56 & 78 & 28 & 190 & 134 & 40 & 80 & 36 & 160 & 152 & 0.74 & 0.89 & 7 \\
\hline Wang et al. (2014) & PCR-RFLP & PE & China & Asian & 232 & 447 & 90 & 90 & 52 & 270 & 194 & 117 & 210 & 120 & 444 & 450 & 0.20 & 0.61 & 7 \\
\hline Langmia et al. (2016) & MassArray & PTB & Malaysia & Asian & 93 & 389 & 26 & 47 & 20 & 99 & 87 & 98 & 199 & 92 & 395 & 383 & 0.64 & 0.89 & 7 \\
\hline Nasr et al. (2017) & PCR-RFLP & $\mathrm{PE}$ & Egypt & Arab & 80 & 80 & 28 & 28 & 24 & 84 & 76 & 20 & 40 & 20 & 80 & 80 & 1.00 & 1.00 & 6 \\
\hline Rahmani et al. (2018) & PCR-RFLP & RSA & Iran & Caucasian & 100 & 100 & 37 & 44 & 19 & 118 & 82 & 38 & 42 & 20 & 118 & 82 & 0.19 & 0.61 & 7 \\
\hline
\end{tabular}

RSA: Recurrent spontaneous abortion; PE: Preeclampsia; PTB: Preterm birth; USA: United States; RT PCR: Real-time PCR. PHWE: The P-value of chi- test for Hardy-Weinberg Equilibrium (HWE) in controls; PHWE: PHWE corrected for multiple testing by FDR method. 
Table 3. Main characteristics of the studies included in the meta-analysis for IL-1 $\beta+3954 \mathrm{C}>\mathrm{T}$

\begin{tabular}{|c|c|c|c|c|c|c|c|c|c|c|c|c|c|c|c|c|c|c|c|}
\hline Study (year) & $\begin{array}{l}\text { Genotyping } \\
\text { method }\end{array}$ & Disease & Country & Ethnicity & Case & Control & & & Cases & & & & & ontrols & & & $P_{\text {HWE }}$ & $\mathrm{P}_{\text {HWE}} *$ & Score \\
\hline IL- $1 \beta,+3954 \mathrm{C}>\mathrm{T}$ & & & & & & & $\mathrm{CC}$ & CT & TT & $\mathrm{C}$ & $\mathrm{T}$ & $\mathrm{CC}$ & CT & TT & $\mathrm{C}$ & $\mathrm{T}$ & & & \\
\hline Reid et al. (2001) & PCR-RFLP & PE & UK & Caucasian & 17 & 40 & 11 & 6 & 0 & 28 & 6 & 26 & 14 & 0 & 66 & 14 & 0.18 & 0.37 & 6 \\
\hline Ma et al. (2011) & PCR-RFLP & RSA & China & Asian & 162 & 156 & 124 & 38 & 0 & 286 & 38 & 130 & 26 & 0 & 286 & 26 & 0.26 & 0.38 & 7 \\
\hline Mohajertehran et al. (2012) & PCR-RFLP & $\mathrm{PE}$ & Iran & Asian & 54 & 50 & 28 & 20 & 6 & 76 & 32 & 28 & 20 & 2 & 76 & 24 & 0.49 & 0.59 & 6 \\
\hline Galvao et al. (2016) & RT PCR & PE & Brazil & Hispanic & 168 & 449 & 5 & 58 & 105 & 68 & 268 & 181 & 87 & 181 & 449 & 449 & 0 & 0 & 7 \\
\hline Langmia et al. (2016) & MassArray & PTB & Malaysia & Asian & 94 & 398 & 83 & 11 & 0 & 177 & 11 & 346 & 52 & 0 & 744 & 52 & 0.16 & 0.38 & 7 \\
\hline Tavakkol Afshari et al. (2016) & PCR-RFLP & PE & Iran & Arab & 153 & 150 & 80 & 57 & 16 & 217 & 89 & 84 & 60 & 6 & 228 & 72 & 0.24 & 0.38 & 7 \\
\hline Wang et al. (2019) & PCR-RFLP & RSA & China & Asian & 598 & 603 & 482 & 111 & 5 & 1075 & 121 & 474 & 121 & 8 & 1069 & 137 & 0.93 & 0.93 & 8 \\
\hline
\end{tabular}

RSA: Recurrent spontaneous abortion; PE: Preeclampsia; PTB: Preterm birth; UK: United Kingdom; RT PCR: Real-time PCR. PHwE: The P-value of chi- test for Hardy-Weinberg Equilibrium (HWE) in controls; PHwE: PHWE corrected for multiple testing by FDR method.

Table 4. The pooled ORs and $95 \%$ CIs for the association between IL1- $\beta$ polymorphisms and overall risk of gestational disorders

\begin{tabular}{|c|c|c|c|c|c|c|c|c|}
\hline 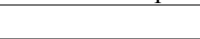 & & & Associat & & & terogeneity & & Egger's test \\
\hline \multirow{2}{*}{\multicolumn{9}{|c|}{ Genetic model }} \\
\hline & & & & & & & & \\
\hline \multirow{5}{*}{ IL $-1 \beta-511 \mathrm{C}>\mathrm{T}$} & 11 & C vs. T & $1.07(0.91-1.26)$ & 0.39 & Random & 0.0001 & $70.44 \%$ & 0.0002 \\
\hline & 11 & CT vs. TT & $0.90(0.78-1.04)$ & 0.14 & Fixed & 0.32 & $31.32 \%$ & 0.15 \\
\hline & 11 & CC vs. TT & $1.15(0.85-1.58)$ & 0.39 & Random & 0.00 & $68.03 \%$ & 0.0005 \\
\hline & 11 & $\mathrm{CT}+\mathrm{CC}$ vs. TT & $1.00(0.80-1.25)$ & 0.98 & Random & 0.03 & $57.88 \%$ & 0.008 \\
\hline & 11 & $\mathrm{CC}$ vs. $\mathrm{CT}+\mathrm{TT}$ & $1.18(0.95-1.46)$ & 0.12 & Random & 0.0004 & $56.10 \%$ & 0.011 \\
\hline \multirow[t]{5}{*}{$\mathrm{IL}-1 \beta-31 \mathrm{~T}>\mathrm{C}$} & 5 & T vs. C & $0.89(0.71-1.11)$ & 0.31 & Random & 0.04 & $60.10 \%$ & 0.12 \\
\hline & 5 & CT vs. CC & $0.74(0.59-0.92)$ & 0.01 & Fixed & 0.30 & $17.30 \%$ & 0.01 \\
\hline & 5 & TT vs. CC & $0.80(0.54-1.20)$ & 0.28 & Random & 0.08 & $51.94 \%$ & 0.23 \\
\hline & 5 & $\mathrm{CT}+\mathrm{TT}$ vs. $\mathrm{CC}$ & $0.74(0.60-0.91)$ & 0.01 & Fixed & 0.13 & $44.41 \%$ & 0.02 \\
\hline & 5 & TT vs. $\mathrm{CT}+\mathrm{CC}$ & $0.92(0.73-1.15)$ & 0.47 & Fixed & 0.16 & $39.31 \%$ & 0.64 \\
\hline \multirow{5}{*}{ IL- $\beta+3954 C>T$} & 5 & T vs. C & $1.44(0.75-2.77)$ & 0.27 & Random & 0.0001 & $93.35 \%$ & 0.88 \\
\hline & 5 & CT vs. CC & $1.79(0.82-3.91)$ & 0.14 & Random & 0.0001 & $90.95 \%$ & 0.17 \\
\hline & 3 & TT vs. CC & $3.37(0.46-24.88)$ & 0.23 & Random & 0.0001 & $91.53 \%$ & 0.72 \\
\hline & 5 & $\mathrm{CT}+\mathrm{TT}$ vs. $\mathrm{CC}$ & $1.82(0.84-3.95)$ & 0.13 & Random & 0.0001 & $91.18 \%$ & 0.16 \\
\hline & 3 & TT vs. $\mathrm{CT}+\mathrm{CC}$ & $1.83(0.86-3.90)$ & 0.12 & Random & 0.07 & $63.05 \%$ & 0.54 \\
\hline
\end{tabular}

$\mathrm{P}<0.05$ is considered as statistically significant (bolded P-value). 


\begin{tabular}{|c|c|c|c|c|c|c|c|c|c|c|c|c|c|c|c|c|}
\hline & No & $\begin{array}{c}\mathrm{OR} \\
(95 \% \mathrm{CI})\end{array}$ & $\mathrm{P}$ & $\mathrm{I}^{2}(\%)$ & $\begin{array}{c}\mathrm{OR} \\
(95 \% \mathrm{CI})\end{array}$ & $\mathrm{P}$ & $\mathrm{I}^{2}(\%)$ & $\begin{array}{c}\text { OR } \\
(95 \% \mathrm{CI})\end{array}$ & $\mathrm{P}$ & $\mathrm{I}^{2}(\%)$ & $\begin{array}{c}\mathrm{OR} \\
(95 \% \mathrm{CI})\end{array}$ & $P$ & $\mathrm{I}^{2}(\%)$ & $\begin{array}{c}\text { OR } \\
(95 \% \mathrm{CI})\end{array}$ & $\mathrm{P}$ & $I^{2}(\%)$ \\
\hline$-511 \mathrm{C}>\mathrm{T}$ & & \multicolumn{3}{|c|}{ C vs. T } & \multicolumn{3}{|c|}{ CT vs. TT } & \multicolumn{3}{|c|}{ CC vs. TT } & \multicolumn{3}{|c|}{ CT $+\mathrm{CC}$ vs. TT } & \multicolumn{3}{|c|}{ CC vs. CT+TT } \\
\hline $\begin{array}{l}\text { Type } \\
\text { PTB }\end{array}$ & 4 & $\begin{array}{c}1.09 \\
(0.97-1.27)\end{array}$ & 0.16 & 0 & $\begin{array}{c}0.84 \\
(0.66-106)\end{array}$ & 0.15 & 0 & $\begin{array}{c}1.15 \\
(0.90-148)\end{array}$ & 0.26 & 0 & $\begin{array}{c}0.95 \\
(077-119)\end{array}$ & 0.68 & 0 & $\begin{array}{c}1.29 \\
(1.06-1.58)\end{array}$ & 0.01 & 6.35 \\
\hline RSA & 5 & $\begin{array}{c}1.12 \\
(0.84-1.52)\end{array}$ & 0.44 & 78.05 & $\begin{array}{c}1.04 \\
(0.84-1.30)\end{array}$ & 0.7 & 15.88 & $\begin{array}{c}1.26 \\
(0.68-2.35)\end{array}$ & 0.46 & 76.02 & $\begin{array}{c}1.10 \\
(0.76-1.59)\end{array}$ & 0.62 & 62.80 & $\begin{array}{c}1.24 \\
(0.80-1.93)\end{array}$ & 0.33 & 68.27 \\
\hline $\begin{array}{l}\text { Ethnicity } \\
\text { Asian }\end{array}$ & 7 & $\begin{array}{c}1.00 \\
(0.84-1.20)\end{array}$ & 0.96 & 70.28 & $\begin{array}{c}0.85 \\
(0.72-0.99)\end{array}$ & 0.036 & 32.64 & $\begin{array}{c}0.99 \\
(0.69-1.42)\end{array}$ & 0.96 & 68.68 & $\begin{array}{c}0.90 \\
(0.71-1.13)\end{array}$ & 0.36 & 56.69 & $\begin{array}{c}1.11 \\
(0.85-1.45)\end{array}$ & 0.42 & 60.39 \\
\hline Caucasian & 3 & $\begin{array}{c}0.80 \\
(0.50-1.26) \\
\end{array}$ & 0.33 & 78.75 & $\begin{array}{c}1.26 \\
(0.83-1.93) \\
\end{array}$ & 0.27 & 0 & $\begin{array}{c}1.56 \\
(0.67-3.61) \\
\end{array}$ & 0.3 & 73.51 & $\begin{array}{c}1.40 \\
(0.75-2.59) \\
\end{array}$ & 0.28 & 58.91 & $\begin{array}{c}1.29 \\
(0.75-2.34) \\
\end{array}$ & 0.36 & 69.79 \\
\hline$-31 \mathrm{~T}>\mathrm{C}$ & & \multicolumn{3}{|c|}{ T vs. C } & \multicolumn{3}{|c|}{ CT vs. CC } & \multicolumn{3}{|c|}{ TT vs. CC } & \multicolumn{3}{|c|}{ CT+TT vs. CC } & \multicolumn{3}{|c|}{ TT vs. $\mathrm{CT}+\mathrm{CC}$} \\
\hline $\begin{array}{l}\text { Type } \\
\text { PTB }\end{array}$ & 2 & $\begin{array}{c}1.08 \\
(0.85-1.37)\end{array}$ & 0.55 & 59.71 & $\begin{array}{c}0.92 \\
(0.61-1.39)\end{array}$ & 0.70 & 0 & $\begin{array}{c}1.15 \\
(0.72-1.83)\end{array}$ & 0.57 & 53.67 & $\begin{array}{c}0.99 \\
(0.68-1.46)\end{array}$ & 0.98 & 0 & $\begin{array}{c}1.23 \\
(0.65-2.31)\end{array}$ & 0.53 & 63.56 \\
\hline RSA & 2 & $\begin{array}{c}0.83 \\
(0.65-1.06)\end{array}$ & 0.14 & 24.62 & $\begin{array}{c}0.83 \\
(0.56-1.23)\end{array}$ & 0.36 & 11.18 & $\begin{array}{c}0.70 \\
(0.43-1.14)\end{array}$ & 0.15 & 17.29 & $\begin{array}{c}0.79 \\
(0.55-1.15)\end{array}$ & 0.22 & 33.59 & $\begin{array}{c}0.78 \\
(0.51-1.20)\end{array}$ & 0.26 & 0 \\
\hline $\begin{array}{l}\text { Ethnicity } \\
\text { Asian }\end{array}$ & 4 & $\begin{array}{c}0.87 \\
(0.67-1.14)\end{array}$ & 0.32 & 67.87 & $\begin{array}{c}0.70 \\
(0.55-0.89)\end{array}$ & 0.003 & 6.10 & $\begin{array}{c}0.78 \\
(0.48-1.25)\end{array}$ & 0.30 & 61.71 & $\begin{array}{c}0.70 \\
(0.56-0.88)\end{array}$ & 0.002 & 46.60 & $\begin{array}{c}0.94 \\
(0.65-1.35)\end{array}$ & 0.74 & 54.45 \\
\hline
\end{tabular}

$\mathrm{P}<0.05$ is considered as statistically significant (bolded P-value). 


\section{IL-16 -31T>C, CT vs. CC}

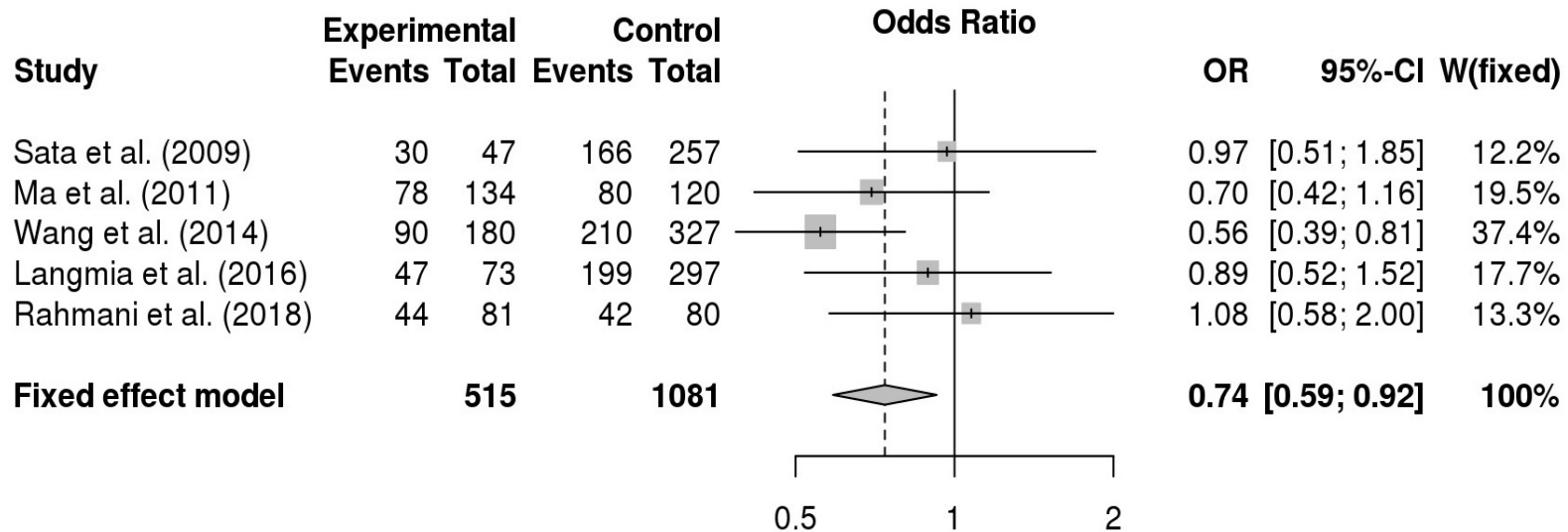

Fig. 2. Forest plot describing the meta-analysis for the association between the IL-1 $\beta-31 \mathrm{~T}>\mathrm{C}$ polymorphism and overall risk of gestational disorders under the codominant CT vs. CC contrasted model.

Meta-analysis results of IL1-6 +3954C > T polymorphism

An overall analysis of 5 studies for IL1- $\beta+3954 C>$ T polymorphisms, including 1175 cases and 1756 controls, revealed no significant association between the SNP and risk of gestational disorders (Table 4). Unfortunately, we could not carry out the subgroup analysis for this variant due to low genotypic frequencies.

\section{Heterogeneity and publication bias}

The Egger's test showed significant publication bias regarding $-511 \mathrm{C}>\mathrm{T}$ and $-31 \mathrm{~T}>\mathrm{C}$ polymorphisms in some studied models (Table 4). Except for the codominant model of $-511 \mathrm{C}>\mathrm{T}$ and recessive model of $+3954 \mathrm{C}>\mathrm{T}$ polymorphisms, high degrees of heterogeneity was found between studies under the assessed genetic models of both SNPs $(p<0.05)$. As regards -31 polymorphism, heterogeneity was observed between studies under the allelic mod$\mathrm{el}(\mathrm{p}=0.04)$.

\section{Sensitivity analysis}

To examine the effect of each study on summary ORs, a sensitivity analysis was carried out by deleting each study one by one in all inheritance modes. We found that ORs were not statistically influenced, which proved the accuracy of pooled results. Figure 3 shows the sensitivity analysis describing the correlation between the IL-1 $\beta-31 \mathrm{~T}>\mathrm{C}$ polymorphism and the risk of gestational disorders under the codominant CT vs. CC genetic model.

\section{IL-16 -31T>C , CT vs. CC}

\section{Study}

Omitting Sata et al. (2009)

Omitting Ma et al. (2011)

Omitting Wang et al. (2014)

Omitting Langmia et al. (2016)

Omitting Rahmani et al. (2018)

\section{Fixed effect model}

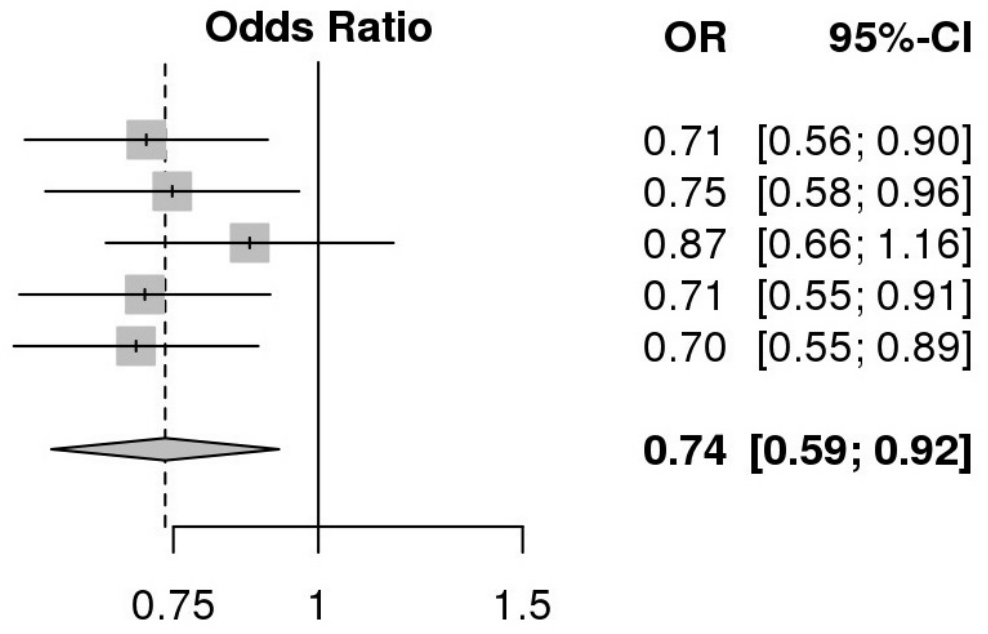

Fig. 3. Sensitivity analysis was carried out to test the effect of the each dataset on the summary ORs between the IL-1 $\beta-31 \mathrm{~T}>\mathrm{C}$ polymorphism and risk of gestational disorders under the codominant CT vs. CC genetic model. 


\section{Discussion}

Immune imbalance leads to unsuccessful pregnancy (40). Aberrant function and expression of cytokines have a potential role in different pregnancy complications (41). The incidence of these complications is significantly higher in developing countries (42).

It has been well documented that polymorphisms could alter cytokine production levels and the strength of cytokine responses (43). The IL- $1 \alpha$ and IL- $1 \beta$, most-studied interleukins in pregnancy disorders, are proinflammatory cytokines that bind to the IL-1 receptor to rapidly initiate signal transduction and apply biological effects (44). The expression of the IL-1 cytokine family by the placenta in normotensive pregnancies highlights the role of this cytokine in pregnancy (45). A wealth of studies have reported the increased level of IL-1 $\beta$ in plasma of women with pregnancy disorders (29). Previous studies have examined the effect of IL1- $\beta$ gene polymorphisms on gestational disorders. However, the reports for the association between $\quad-511 \mathrm{C}>\mathrm{T}, \quad-31 \mathrm{~T}>\mathrm{C}, \quad$ and $+3954 \mathrm{C}>\mathrm{T}$ polymorphisms, as common IL1 $\beta$ SNPs, and various gestational disorders have shown inconsistent results in different populations. A meta-analysis of available data presents the precise estimation of the effects of the polymorphisms. Previous meta-analyses showed that IL1 $\beta$ polymorphisms affect the risk of various clinical conditions and cancers (46-48). However, to this date, no metaanalysis study has reported a precise correlation between IL1 $\beta$ polymorphisms and the risk of gestational disorders. Hence, in the current study, we combined data from the 13 genetic association studies regarding $-511 \mathrm{C}>\mathrm{T},-31 \mathrm{~T}>\mathrm{C}$, and $+3954 \mathrm{C}>\mathrm{T}$ polymorphisms and the risk of gestational disorders.

Pooled results from our meta-analysis revealed that IL-1 $\beta-31 \mathrm{~T}>\mathrm{C}$ polymorphism reduces the risk of gestational disorders under codominant $\mathrm{CT}$ vs. $\mathrm{CC}$ and dominant $\mathrm{CT}+\mathrm{TT}$ vs. CC genetic models. Besides, in the overall analysis, no noteworthy association was noticed between $-511 \mathrm{C}>\mathrm{T}$ and $+3954 \mathrm{C}>\mathrm{T}$ polymorphisms and the incidence of gestational disorders. By performing the stratified analysis by disease type, our findings showed that $-511 \mathrm{C}>\mathrm{T}$ variant decreased the risk of PTB under the recessive genetic model. Moreover, $+3954 \mathrm{C}>\mathrm{T}$ did not influence the risk of gestational disorders under the assessed genetic models. For counting the potential distracting effect of population admixture, we carried out subgroup analysis by ethnicity. We found the protective effect of $-511 \mathrm{C}>\mathrm{T}$ and $-31 \mathrm{~T}>\mathrm{C}$ polymorphisms against the risk of gestational disorders in Asians.

Awasthi and colleagues carried out a meta-analysis to examine the correlation between IL $1 \beta-511 \mathrm{C} / \mathrm{T}$ polymorphism and the risk of PTB (30). By including four studies (consist of 832 cases and 1101 controls) in the analysis, they failed to find a link between this SNP and vulnerability to PTB under the studied genetic models. Likewise, in the present up-dated meta-analysis, we pooled the results of 11 studies, including 2747 women with gestational disorders and 3436 healthy women, and found the same results. The first meta-analysis describing the correlation between IL1 $\beta$ polymorphisms and risk of RSA was per- formed by Bombell and Mcguire in 2008 (49). By pooling the findings of three case-control studies, they failed to find a noteworthy link between $-511 \mathrm{C} / \mathrm{T}$ and RSA risk, although an association between $-31 \mathrm{~T}$ allele with RSA was detected by pooling trial results of two studies. Another meta-analysis by Agrawal et al. in 2012 revealed similar results for the lack of association between $-511 \mathrm{C} / \mathrm{T}$ and RSA susceptibility (three studies included) (27). In a recent meta-analysis, Zhang et al. pooled five studies (1052 patients and 915 controls). They observed a significant correlation between the $-511 \mathrm{C} / \mathrm{T}$ polymorphism and RSA incidence under the recessive genetic model (50). In our work, however, we found an enhanced risk of PTB under the recessive $\mathrm{CC}$ vs. CT+TT inheritance mode.

IL1 gene polymorphisms served significant roles in the development of gestational disorders. However, the findings of the former meta-analysis were controversial because of the limited sample size. Therefore, updated results can help to get more reliable details of gestational disorders' pathogenesis and find biomarkers for predicting their risk. Our meta-analysis had several limitations. First, gestational disorders are multifactorial, and gene variants cannot be considered the only underlying etiology. Second, we merely examined the role of the polymorphisms while their functional consequences were not evaluated. Third, we did not consider gene-environment interactions and haplotypes due to the unavailability of individual data in retrieved publications. Finally, heterogeneity was observed between studies, which might be due to the varied study designs, application of different genotyping methods, and ethnicity differences.

\section{Conclusion}

In conclusion, our results failed to support an association between two IL-1 $\beta$ polymorphisms, $511 \mathrm{C}>\mathrm{T}$ and $+3954 \mathrm{C}>\mathrm{T}$, with the overall risk of gestational disorders. In contrast, the $31 \mathrm{~T}>\mathrm{C}$ variant reduced the incidence of such diseases. Further studies are encouraged to get more precise estimates of effect sizes.

\section{Acknowledgment}

The authors wish to thank Dr. Alireza Ansari Moghadam for his contribution to data analyses.

\section{Conflict of Interests}

The authors declare that they have no competing interests.

\section{References}

1. Teimoori B, Moradi-shahrebabak M, Razavi M, Rezaei M, HaratiSadegh M, Salimi S. The effect of GPx-1 rs1050450 and MnSOD rs4880 polymorphisms on PE susceptibility: a case-control study. Mol Biol Rep. 2019;46(6):6099-104.

2. Mohammadpour-Gharehbagh A, Eskandari M, Sadegh MH, Nematollahi MH, Rezaei M, Rasouli A, et al. Genetic and epigenetic analysis of the BAX and BCL2 in the placenta of pregnant women complicated by preeclampsia. Apoptosis. 2019;24(3-4):301-11.

3. Holland O, Nitert MD, Gallo LA, Vejzovic M, Fisher JJ, Perkins AV. Placental mitochondrial function and structure in gestational disorders. Placenta. 2017;54:2-9.

4. Harati-Sadegh M, Kohan L, Teimoori B, Mehrabani M, Salimi S. Analysis of polymorphisms, promoter methylation, and mRNA 
expression profile of maternal and placental P53 and P21 genes in preeclamptic and normotensive pregnant women. J Biomed Sci. 2019;26(1):92.

5. Segerer S, Kämmerer U, Kapp M, Dietl J, Rieger L. Upregulation of chemokine and cytokine production during pregnancy. Gynecol Obstet Invest. 2009;67(3):145-50.

6. Harati-Sadegh M, Kohan L, Teimoori B, Mehrabani M, Salimi S. The effects of placental long noncoding RNA H19 polymorphisms and promoter methylation on $\mathrm{H} 19$ expression in association with preeclampsia susceptibility. Iubmb Life. 2020;72(3):413-25.

7. Challis JR, Lockwood CJ, Myatt L, Norman JE, Strauss III JF, Petraglia F. Inflammation and pregnancy. Reprod Sci. 2009;16(2):206-15.

8. Li M, Huang SJ. Innate immunity, coagulation and placenta-related adverse pregnancy outcomes. Thromb Res. 2009;124(6):656-62.

9. Desoye G, Hauguel-de Mouzon S. The human placenta in gestational diabetes mellitus: the insulin and cytokine network. Diabetes Care. 2007;30(Supplement 2):S120-S6.

10. Prins JR, Gomez-Lopez N, Robertson SA. Interleukin-6 in pregnancy and gestational disorders. J Reprod Immunol. 2012;95(12):1-14.

11. Paulesu L, King A, Loke Y, Cintorino M, Bellizzi E, Boraschi D. Immunohistochemical localization of IL-1 alpha and IL-1 beta in normal human placenta. Lymphokine Cytokine Res. 1991;10(6):4438.

12. Wang Z, Yunis E, De los Santos M, Xiao L, Anderson D, Hill J. T helper 1-type immunity to trophoblast antigens in women with a history of recurrent pregnancy loss is associated with polymorphism of the IL1B promoter region. Genes Immun. 2002;3(1):38-42.

13. Kwak-Kim J, Yang KM, Gilman-Sachs A. Recurrent pregnancy loss: a disease of inflammation and coagulation. J Obstet Gynaecol Res. 2009;35(4):609-22.

14. Prakash P, Victor D. Interleukin-1b gene polymorphism and its association with chronic periodontitis in South Indian population. Int J Genet Mol Biol. 2010;2(8):179-83.

15. Zur Hausen A, Crusius JBA, Murillo LS, Alizadeh BZ, Morré SA, Meijer CJ, et al. IL-1B promoter polymorphism and Epstein-Barr virus in Dutch patients with gastric carcinoma. Int $\mathrm{J}$ Cancer. 2003;107(5):866-7.

16. Kim JO, Lee WS, Lee BE, Jeon YJ, Kim YR, Jung SH, et al. Interleukin-1beta-511T> $\mathrm{C}$ genetic variant contributes to recurrent pregnancy loss risk and peripheral natural killer cell proportion. Fertil Steril. 2014;102(1):206-12. e5.

17. Leme Galvão LP, Menezes FE, Mendonca C, Barreto I, AlvimPereira C, Alvim-Pereira F, et al. Analysis of association of clinical aspects and IL1B tagSNPs with severe preeclampsia. Hypertens Pregnancy. 2016;35(1):112-22.

18. Schmid M, Haslinger P, Stary S, Leipold H, Egarter C, Grimm C. Interleukin-1 beta gene polymorphisms and preterm birth. Eur J Obstet Gynecol Reprod Biol. 2012;165(1):33-6.

19. Stang A. Critical evaluation of the Newcastle-Ottawa scale for the assessment of the quality of nonrandomized studies in meta-analyses. Eur J Epidemiol. 2010;25(9):603-5.

20. Martorell-Marugan J, Toro-Dominguez D, Alarcon-Riquelme ME, Carmona-Saez P. MetaGenyo: a web tool for meta-analysis of genetic association studies. BMC Bioinform. 2017;18(1):563.

21. Cummings P. Methods for estimating adjusted risk ratios. Stata J. 2009;9(2):175-96.

22. Hefler LA, Tempfer CB, Bashford MT, Unfried G, Zeillinger R, Schneeberger C, et al. Polymorphisms of the angiotensinogen gene, the endothelial nitric oxide synthase gene, and the interleukin- $1 \beta$ gene promoter in women with idiopathic recurrent miscarriage. Mol Hum Reprod. 2002;8(1):95-100.

23. Wang Z, Yunis E, De los Santos M, Xiao L, Anderson D, Hill J. T helper 1-type immunity to trophoblast antigens in women with a history of recurrent pregnancy loss is associated with polymorphism of the IL1B promoter region. Genes Immun. 2002;3(1):38.

24. Linjawi S, Li T-C, Laird S, Blakemore A. Interleukin-1 receptor antagonist and interleukin-1 beta polymorphisms in women with recurrent miscarriage. Fertil Steril. 2005;83(5):1549-52.

25. Sata F, Toya S, Yamada H, Suzuki K, Saijo Y, Yamazaki A, et al. Proinflammatory cytokine polymorphisms and the risk of preterm birth and low birthweight in a Japanese population. Mol Hum Reprod. 2009;15(2):121-30.

26. Ma X, Xu LJ, Wang J, Xian MM, Liu M. Association of IL-1 $\beta$ and
IL-6 gene polymorphisms with recurrent spontaneous abortion in a Chinese Han population. Int J Immunogenet. 2012;39(1):15-9.

27. Agrawal S, Parveen F, Faridi R, Prakash S. Interleukin-1 gene cluster variants and recurrent pregnancy loss among North Indian women: retrospective study and meta-analysis. Reprod Biomed Online. 2012;24(3):342-51.

28. Yılmaz Y, Verdi H, Taneri A, Yazııı AC, Ecevit AN, Karakaş NM, et al. Maternal-fetal proinflammatory cytokine gene polymorphism and preterm birth. DNA Cell Biol. 2012;31(1):92-7.

29. Wang X, Jiang F, Liang Y, Xu L, Li H, Liu Y, et al. Interleukin1beta-31C/T and $-511 \mathrm{~T} / \mathrm{C}$ polymorphisms were associated with preeclampsia in Chinese Han population. PLoS One. 2014;9(9):e106919.

30. Awasthi S, Pandey M, Sundaram V, Dixit P. Association of TNF- $\alpha$ and IL-1 $\beta$ gene polymorphisms with preterm birth-replication study and meta analysis. Int J Sci Res. 2015;4:1679-85.

31. Langmia IM, Apalasamy YD, Omar SZ, Mohamed Z. Impact of IL1B gene polymorphisms and interleukin 1B levels on susceptibility to spontaneous preterm birth. Pharmacog Genom. 2016;26(11):505-9.

32. Pereyra S, Bertoni B, Sapiro R. Interactions between environmental factors and maternal-fetal genetic variations: strategies to elucidate risks of preterm birth. Eur J Obstet Gynecol Reprod Biol. 2016;202:20-5.

33. Nasr AS, El Azizy HM, Hassan S, Salem H, Diaa N. Interleukin-1ßgene polymorphisms in preeclamptic Egyptian women. Middle East Fertil Soc J. 2017;22(4):285-9.

34. Ali SR, Paknejad Z, Mohammadkhanlou M, Daneshparvar M. Association of of IL-1 receptor antagonist (IL-1RN) and interleukin$1 \beta$ genes (IL-1 $\beta$ ) polymorphisms with recurrent pregnancy loss in Iranian Azeri women. Horm Mol Biol Clin Invest. 2017;33(3).

35. Wang T, Lu N, Cui Y, Tian L. Polymorphisms in interleukin genes and their association with the risk of recurrent pregnancy loss. Genes Genet. 2019;94(3):109-16.

36. Majcher S, Ustianowski P, Tarnowski M, Dziedziejko V, Safranow K, Pawlik A. IL-1 $\beta$ and IL-10 gene polymorphisms in women with gestational diabetes. J Mat Fet Neonat Med. 2019:1-6.

37. Reid JG, Simpson NA, Walker RG, Economidou O, Shillito J, Duffy SR, et al. The carriage of proinflammatory cytokine gene polymorphisms in recurrent pregnancy loss. Am J Reprod Immunol. 2001;45(1):35-40.

38. Mohajertehran F, Afshari JT, Rezaieyazdi Z, Ghomian N. Association of single nucleotide polymorphisms in the human tumor necrosis factor- $\alpha$ and interleukin $1-\beta$ genes in patients with preeclampsia. Iran J Allergy Asthma Immunol. 2012:224-9.

39. Tavakkol Afshari Z, Rahimi HR, Ehteshamfar SM, Ganjali R, Tara F, Shapouri Moghadam A. Tumor necrosis factor- $\alpha$ and interleukin-1$\beta$ polymorphisms in preeclampsia. Iran J Immunol. 2016;13(4):30916.

40. Kwak-Kim J, Park JC, Ahn HK, Kim JW, Gilman-Sachs A. Immunological modes of pregnancy loss. Am J Reprod Immunol. 2010;63(6):611-23.

41. Yockey LJ, Iwasaki A. Interferons and proinflammatory cytokines in pregnancy and fetal development. Immunity. 2018;49(3):397-412.

42. Menon R. Spontaneous preterm birth, a clinical dilemma: etiologic, pathophysiologic and genetic heterogeneities and racial disparity. Acta Obstet Gynecol Scand. 2008;87(6):590-600.

43. Razdaibiedina A, Khobzey M, Tkachenko V, Vorobiova I. Effects of single-nucleotide polymorphisms in cytokine, Toll-like receptor, and progesterone receptor genes on risk of miscarriage. Obstet Gynecol Int. 2018;2018.

44. Li J, Liu M, Zong J, Tan P, Wang J, Wang X, et al. Genetic variations in IL1A and IL1RN are associated with the risk of preeclampsia in Chinese Han population. Sci Rep. 2014;4(1):1-6.

45. Southcombe J, Redman C, Sargent I, Granne I. Interleukin-1 family cytokines and their regulatory proteins in normal pregnancy and preeclampsia. Clin Exp Immunol. 2015;181(3):480-90.

46. Wang Y, Liu L, Xu X, Wang J, Zhou H, Li T, et al. Association of interleukin-1 $\beta-511 \mathrm{C} / \mathrm{T}$ promoter polymorphism with COPD risk: a meta-analysis. Genet Mol Res. 2015;14(2):4477-84.

47. Sun X, Cai H, Li Z, Li S, Yin W, Dong G, et al. Association between IL-1 $\beta$ polymorphisms and gastritis risk: A meta-analysis. Medicine. 2017;96(5).

48. Chen B, Luo M-x, Zhou X, Lv Y, Su G-q. Correlation between interleukin-1 $\beta-511 \mathrm{C} / \mathrm{T}$ polymorphism and gastric cancer in Chinese populations: a meta-analysis. Medical science monitor. Med Sci Mon 
Int Med J Exp Clin Res. 2016;22:1742.

49. Bombell S, Mcguire W. Cytokine polymorphisms in women with recurrent pregnancy loss: meta-analysis. Aust N Z J Obstet Gynaecol. 2008;48(2):147-54.

50. Zhang M, Xu J, Bao X, Niu W, Wang L, Du L, et al. Association between genetic polymorphisms in interleukin genes and recurrent pregnancy loss-a systematic review and meta-analysis. PloS One. $2017 ; 12(1)$ 